\title{
Biogeography of marine giant viruses reveals their interplay with eukaryotes and ecological functions
}

\section{$\operatorname{AUTHOR}(\mathrm{S})$ :}

Endo, Hisashi; Blanc-Mathieu, Romain; Li, Yanze; Salazar, Guillem; Henry, Nicolas; Labadie, Karine; de Vargas, Colomban; ... Karp-Boss, Lee; Sunagawa, Shinichi; Ogata, Hiroyuki

\section{CITATION:}

Endo, Hisashi ... [et al]. Biogeography of marine giant viruses reveals their interplay with eukaryotes and ecological functions. Nature Ecology \& Evolution 2020, 4(12): 1639-1649

\section{ISSUE DATE:}

2020-12

URL:

http://hdl.handle.net/2433/259797

\section{RIGHT:}

This is a post-peer-review, pre-copyedit version of an article published in 'Nature Ecology \& Evolution'. The final authenticated version is available online at: https://doi.org/10.1038/s41559-020-01288-w.; The full-text file will be made open to the public on 07 September 2021 in accordance with publisher's 'Terms and Conditions for Self-Archiving'; この 論文は出版社版でありません。引用の際には出版社版をご確認ご利用ください。; This is not the published version.

Please cite only the published version. 


\section{Biogeography of marine giant viruses reveals their interplay} 2 with eukaryotes and ecological functions

4 Hisashi Endo ${ }^{1}$, Romain Blanc-Mathieu ${ }^{1,2}$, Yanze Li ${ }^{1}$, Guillem Salazar ${ }^{3}$, Nicolas Henry ${ }^{4,5}$,

5 Karine Labadie ${ }^{6}$, Colomban de $\operatorname{Vargas}^{4,5}$, Matthew B. Sullivan ${ }^{7,8}$, Chris Bowler ${ }^{9,10}$, 6 Patrick Wincker ${ }^{10,11}$, Lee Karp-Boss ${ }^{12}$, Shinichi Sunagawa ${ }^{3}$, Hiroyuki Ogata ${ }^{1, *}$

8 Affiliations:

9 1. Bioinformatics Center, Institute for Chemical Research, Kyoto University, Gokasho, Uji, Kyoto, 611-0011, Japan

2. Laboratoire de Physiologie Cellulaire \& Végétale, CEA, Univ. Grenoble Alpes, CNRS, INRA, IRIG, Grenoble, France

3. Department of Biology, Institute of Microbiology and Swiss Institute of Bioinformatics, ETH Zürich, Zürich 8093, Switzerland

4. CNRS, UMR 7144, Station Biologique de Roscoff, Place Georges Teissier, 29680 Roscoff, France.

5. Sorbonne Universités, UPMC Université Paris 06, UMR 7144, Station Biologique de Roscoff, Place Georges Teissier, 29680 Roscoff, France.

6. Genoscope, Institut de Biologie François-Jacob, Commissariat à l'Énergie Atomique (CEA), Université Paris-Saclay, Évry, France.

7. Department of Microbiology, The Ohio State University, Columbus, OH 43210, USA

8. Department of Civil, Environmental and Geodetic Engineering, The Ohio State University, Columbus, OH 43210, USA

9. Institut de Biologie de l'ENS (IBENS), Département de biologie, École normale supérieure, CNRS, INSERM, Université PSL, Paris 75005, France

10. Research Federation for the study of Global Ocean Systems Ecology and Evolution, FR2022/Tara Oceans GOSEE, 3 rue Michel-Ange, 75016 Paris, France

11. Génomique Métabolique, Genoscope, Institut de Biologie François Jacob,

30 Commissariat à l'Énergie Atomique (CEA), CNRS, Université Évry, Université 31 Paris-Saclay, Évry, France. 
32 12. School of Marine Sciences, University of Maine, Orono, ME, USA 33

34 *Corresponding author:

35 H. Ogata, E-mail: ogata@kuicr. kyoto-u.ac.jp, Phone: +81-774-38-3270 36 


\section{Abstract}

38 Nucleocytoplasmic large DNA viruses (NCLDVs) are ubiquitous in marine 39 environments and infect diverse eukaryotes. However, little is known about their 40 biogeography and ecology in the ocean. By leveraging the Tara Oceans pole-to-pole metagenomic data set, we investigated the distribution of NCLDVs across size fractions, depths and biomes, as well as their associations with eukaryotic communities. Our analyses revealed a heterogeneous distribution of NCLDVs across oceans, with an

44 elevated uniqueness in polar biomes. The community structures of NCLDV families were correlated with specific eukaryotic lineages including many photosynthetic groups.

46 NCDLV communities were generally distinct between surface and mesopelagic zones, 47 but at some locations, they exhibited a high similarity between the two depths. This 48 vertical similarity was correlated to surface phytoplankton biomass but not to physical 49 mixing processes, suggesting the potential role of vertical export in structuring mesopelagic NCLDV communities. These results underscore the importance of the coupling between NCLDVs and eukaryotes in biogeochemical processes in the ocean. 


\section{Introduction}

55 The photic zone is the most productive layer of the ocean, containing a wide variety 56 of microorganisms such as bacteria, autotrophic and heterotrophic protists and 57 multicellular organisms. The population dynamics of these organisms determine the 58 flows of energy and materials through marine food webs, playing a fundamental role in 59 ecosystem functioning and biogeochemical cycles in the ocean ${ }^{1,2}$. Viruses exert a topdown control on marine organisms and release material to the pools of particulate and

61 dissolved organic matter ${ }^{3}$. This material and remineralized inorganic nutrients are utilized 62 by autotrophic and mixotrophic phytoplankton ${ }^{4}$. The recycling of nutrients in the surface 63 layer potentially reduces the transfer of fixed organic carbon to higher trophic levels and 64 the deep sea ${ }^{5,6}$. However, it is also possible that viruses enhance downward carbon flux by facilitating cell aggregation and producing carbon-enriched materials from infected 66 cells $^{7-9}$.

67 Nucleocytoplasmic large DNA viruses (NCLDVs or so-called "giant viruses”) 68 represent a monophyletic group of viruses that infect a variety of eukaryotic lineages ${ }^{10-12}$. 69 Studies focusing on conserved marker genes such as family B DNA polymerase (polB) 70 have revealed that NCLDVs are highly diverse and abundant in aquatic environments ${ }^{13-}$ 16. The diversity of a family of NCLDVs, namely Mimiviridae, exceeds that of bacteria

72 and archaea in the ocean ${ }^{17}$ and their richness in a few liters of seawater can reach more 73 than 5,000 operational taxonomic units ${ }^{18}$. More recently, several thousand draft genomes 74 (i.e., metagenome-assembled genomes; MAGs) of NCLDVs were constructed from environmental sequences, thanks to the development of high-throughput sequencing and bioinformatics technologies ${ }^{19,20}$. However, the global biogeography of marine NCLDVs still remains under-explored.

A growing number of marine eukaryotes have been reported as host organisms of 79 NCLDVs, particularly phytoplankton groups such as haptophytes, chlorophytes and 80 dinoflagellates ${ }^{21-23}$. Other eukaryotic lineages, including non-photosynthetic organisms such as bicosoecids and choanoflagellates, have also been reported as host organisms of 
82 NCLDVs in marine environments ${ }^{24,25}$. These studies collectively suggest the ecological

83 importance of NCLDVs in the ocean via top-down effects on eukaryotic communities.

84 However, our knowledge of NCLDV-host relationships is highly limited, given the large

85 phylogenetic diversities of NCLDVs and microeukaryotes.

86 Here we reveal patterns in the global biogeography of NCLDVs using the

87 metagenomic data from the Tara Oceans project. The metagenomic data cover varying geographic regions including polar and deep-sea ecosystems, in which NCLDVs are under-researched ${ }^{26-28}$. We constructed NCLDV taxonomic abundance profiles for 283 samples, representing two viral size fractions, three ocean depth ranges (surface, deep chlorophyll maximum and mesopelagic), and four biomes (coastal, trades, westerlies and polar). The global biogeography of NCLDVs derived from these data reveals strong associations between NCLDVs and eukaryotic microorganisms. Furthermore, vertical

94 connectivity of NCLDV communities indicates a possible mechanism for how mesopelagic NCLDV communities are structured with respect to ocean biogeochemical processes.

\section{Results}

\section{NCLDV phylotypes detected in Tara Oceans metagenomes}

100 We detected 6,818 PolBs affiliated with NCLDVs in the second version of the Ocean

101 Microbial Reference Gene Catalog (OM-RGC.v2) ${ }^{28}$ using the pplacer phylogenetic 102 placement method ${ }^{29}$ (see methods for details). The OM-RGC.v2 was built based on 370

103 Tara Oceans metagenomes from femto- (<0.2 $\mu$ m; 151 samples), pico- $(0.22-1.6$ or $0.22-$ $1043.0 \mu \mathrm{m}$; 180 samples) and other (39 samples) size fractions. After removing 32 samples 105 with a low NCLDV frequency and 55 samples from non-target size fractions and depths, 106 the remaining 283 samples contained 6,783 NCLDV PolB sequences. The pplacer 107 classified these PolBs into nine NCLDV families/lineages. The number of phylotypes 108 (distinct polB at 95\% nucleotide sequence identity) was the largest in Mimiviridae (5,091 phylotypes), followed by Phycodnaviridae (981 phylotypes). The number of phylotypes 
110 taxonomically assigned to Iridoviridae, Medusavirus and Asfarviridae, were 239, 120

111 and 109, respectively. We also detected PolBs assigned to Pithoviridae (93), Ascoviridae

112 (78), Poxviridae (51) and Marseilleviridae (21). However, Poxviridae was omitted from

113 our discussion as the environmental gene sequences were distantly related to known

114 Poxviridae. Rarefaction analysis showed that, at the end of sampling, the number of

115 NCLDV phylotypes increased by less than $0.01 \%$ per sample for all samples, and ranged

116 from $0.02 \%$ to $0.32 \%$ when samples were divided into different size fractions, depths and

117 biomes (Extended Data Fig. 1).

118 To examine detailed phylogenetic affiliation and to visualize the dispersal 119 characteristics of each NCLDV phylotypes detected by pplacer, we constructed a 120 phylogenetic tree using selected PolB sequences (Extended Data Figs. 2-4). Among the

121 Mimiviridae family, genes closely related to the algal-infecting subfamily, recently 122 proposed as "Mesomimivirinae” (e.g., AaV, CeV, pkV, PgV, PoV and TetV) ${ }^{30}$, which 123 infect pelagophytes (the genus Aureococcus), haptophytes (the genera Haptolina,

124 Prymnesium and Phaeocystis), and chlorophytes (the genera Pyramimonas and 125 Tetraselmis), were relatively abundant. On the other hand, only a few sequences were 126 affiliated with the subfamilies "Megamimivirinae” and "Klosneuvirinae” except the 127 Cafeteria roenbergensis virus (CroV), which is the only member of "Megamimivirinae" 128 isolated from the marine environment ${ }^{24}$. Among Phycodnaviridae, the genus 129 Prasinovirus (e.g., BpV, MpV, OtV and OlV), which infect chlorophyte genera such as 130 Bathycoccus, Micromonas and Ostreococcus, showed the highest richness.

\section{Heterogeneity in NCLDV community structure across size, depth and biomes}

133 The dominant NCLDV taxa detected from all sample locations and depths in the pico134 size fraction were Mimiviridae and Phycodnaviridae, with average contributions of $13564.6 \%$ and 25.4\%, respectively (Fig. 1A). The dominant groups of NCLDVs varied 136 widely among sites and depths in samples from the femto-size fraction (Fig. 1B). In this 137 fraction, Phycodnaviridae and Asfarviridae had relatively high contributions to the total 
138 NCLDVs with the mean values of $29.7 \%$ and $19.9 \%$, respectively. Mimiviridae and

139 Ascoviridae were also important contributors with mean values of $12.2 \%$ and $11.1 \%$, 140 respectively.

141 A non-metric multidimensional scaling (NMDS) analysis showed that NCLDV assemblages clustered according to size fraction, depth and biome (Fig. 2A-2C).

143 Significant differences in NCLDV community composition were detected among all categories (PERMANOVA, $p<0.01$ ), and size fraction, depth and biome explained 5.5\%, $4.3 \%$ and $10.9 \%$ of the total variance, respectively.

146 Taxonomic richness (i.e., number of phylotypes) and Shannon's diversity index were

147 used to investigate variation in NCLDV community diversity. In this study, we analyzed

148 the samples from all depths and size fractions to compare diversity differences among 149 depth ranges, although latitudinal trend in Shannon's diversity for pico-sized 150 communities from the surface was reported previously ${ }^{31}$. In the pico-size fraction, mean 151 values for NCLDV richness at the surface and in the DCM layer were about 1.7 times 152 higher than that in the mesopelagic layer (Kruskal-Wallis and Dunn's post hoc test, $p$ 153 $<0.01$ ) (Extended Data Fig. 5A). In the femto-size fraction, NCLDV richness was significantly higher at the surface and MES layer than in the DCM layer (Dunn's test, $p$ $=0.04-0.05$ ), although the differences were small and not consistent with the pico-size

156 fraction.

\section{High uniqueness of NCLDV phylotypes in the Arctic Ocean}

We analyzed the overlap and uniqueness of NCLDV phylotypes across different ecological zones (i.e., size fraction, depth and biome) to evaluate their ability to disperse across different environments. Each ecological category was divided into two major groups (i.e., pico- and femto-sizes, euphotic and mesopelagic zones, and polar and non-

163 polar biomes), because the NCLDV community in mesopelagic zone or polar biome was 164 separated most significantly from other depths or biomes (Fig. 2). We found 4,003 (59.0\% to the total NCLDVs) shared NCLDV phylotypes across size fractions, 4,737 (69.8\%) 
166 shared phylotypes across depth ranges, and 1,950 (28.7\%) shared phylotypes across

167 biomes (Fig. 3A). Only twelve unique phylotypes were detected in the femto-size fraction,

168 whereas 2,768 unique phylotypes were identified in the pico-size fraction. The euphotic

169 zone (surface and DCM) harbored 1,986 unique phylotypes, whereas the aphotic 170 mesopelagic zone had only 60 unique phylotypes. The polar biome (the Arctic and the

171 Southern Ocean) included 620 unique NCLDV phylotypes, whereas 4,213 unique

172 NCLDVs were detected in non-polar biomes (i.e., trades, westerlies and coastal).

173 To further characterize regional differences in the NCLDV community, we 174 investigated the total and unique NCLDV phylotypes observed in nine geographic regions 175 and the phylotypes shared among regions. The total number of phylotypes was relatively 176 high in the Atlantic, Pacific and Indian Oceans and in the Mediterranean Sea, with values 177 of between 3,665 and 4,685 (Fig. 3B). Lower numbers of NCLDV phylotypes were 178 identified from the Red Sea $(2,653)$ and the Arctic Ocean $(2,467)$. The Southern Ocean presented the lowest number of NCLDV phylotypes (561), although this was based on only 5 samples. The Arctic Ocean samples displayed a high number of unique NCLDV phylotypes (551), which corresponded to $22.3 \%$ of the total phylotypes detected in this region. In contrast, the number of unique phylotypes from other regions ranged from 0 to $134(0.0 \%$ to $3.4 \%)$.

184 There was no linear or saturation trend in the number of total or unique NCLDV 185 phylotypes with increasing sample size (Fig. 3C). The high proportion of unique phylotypes in the Arctic Ocean was not a function of sample size, although the number of total phylotypes detected in the Southern Ocean may be limited by the low number of samples. The phylogenetic positions of unique NCLDVs from the polar biome were dispersed across most of the NCLDV families (Fig. 4)

\section{NCLDV distributions correlate with eukaryotic communities}

192 A partial Mantel test was conducted to assess community associations among the 193 NCLDV families/lineages and major eukaryotic lineages. The pairwise partial correlation 
194 coefficients (Spearman's $\rho$ ) varied from -0.17 to 0.76 (Fig. 5A), and $93.6 \%$ of the 195 examined pairs (225 out of 234 for the pico-size fraction and 213 out of 234 for the femto196 size fraction) showed statistically significant correlations ( $p<0.01$, permutation test) after 197 false discovery rate (FDR) correction. Pairs from pico-sized NCLDV communities with 198 a correlation coefficient $\geq 0.53$ were considered to represent strong positive associations, 199 because 8 out of 9 known marine virus-host lineage associations were recovered by this 200 criterion (Figs. 5A and 5B). Using this threshold, 30 out of 234 NCLDV-eukaryote 201 lineage pairs were found to have strong linkages (Fig. 5C). The NCLDV families/lineages 202 were generally highly correlated with the known host groups among autotrophic and 203 mixotrophic microalgae (haptophytes, chlorophytes, dinophytes, pelagophytes and 204 raphidophytes) ( $\rho=0.54-0.67)$. Interestingly, Mimiviridae was strongly correlated with 205 chrysophyte microalgae $(\rho=0.65)$, which are not currently known as NCLDV hosts. 206 Other than algal lineages, a strong positive correlation was found between Mimiviridae 207 and heterotrophic eukaryote choanoflagellates $(\rho=0.76)$, which are a known lineage of 208 Mimiviridae. A group of non-photosynthetic heterokonts bicosoecids are also a known 209 host of the Mimiviridae species CroV in marine environments, but this group was not 210 highly correlated with Mimiviridae $(\rho=0.30)$.

\section{Potential chrysophyte viruses constitute novel clades of Mimiviridae}

213 To explore possible associations between NCLDVs and chrysophytes as indicated by 214 the Mantel's regression analysis (Fig. 5C), we tested for chrysophyte-derived genes in 215 the metagenome-assembled genomes (MAGs) of NCLDVs generated by Schultz et al. $216(2020)^{19}$ and Moniruzzaman et al. $(2020)^{20}$. The results showed that 89 (82 after removing 217 redundancy) out of 2,263 MAGs contained genes closely related to the transcripts of the 218 chrysophytes (Supplementary Data 1). Comparisons between PolB sequences revealed 21927 PolBs from the OM-RGC.v2 that were closely related to the NCLDV MAGs with 220 chrysophyte homologs. Most of these PolBs constituted novel clades within the branches of Mimiviridae (Fig. 4; Extended Data Fig. 4). We confirmed that other genes in the 
222 contigs that contained chrysophyte homologs are highly similar to the Mimiviridae or

223 Phycodnaviridae sequences in many cases (Extended Data Fig. 6).

\section{Vertical connectivity of NCLDV communities}

The vertical connectivity of NCLDV communities was investigated using Bray-Curtis community similarity measures to compare between epipelagic (surface or DCM) and mesopelagic samples at individual sampling locations. The Bray-Curtis similarities were less than 0.10 for about half of the tested locations (20 out of 36 surface sites and 13 out of 26 DCM sites; Fig. 6A; Extended Data Fig. 7A). All sites in the Arctic Ocean and several sites in tropical and subtropical regions showed relatively high similarities between the two depth $(0.15$ to 0.60$)$. The NCLDV community similarity value was positively correlated with the chlorophyll $a$ concentration in the epipelagic layer

234 (Spearman's $\rho=0.52, p<0.01$, asymptotic $t$ approximation, $\mathrm{n}=36$ for surface; $\rho=0.44$, $p=0.02, \mathrm{n}=25$ for DCM) and NCLDV richness in the mesopelagic layer $(\rho=0.82, p$ $<0.01, \mathrm{n}=36$ for surface; $\rho=0.70, p<0.01, \mathrm{n}=26$ for DCM) (Figs. 6B and 6C; Extended Data Figs. 7B and 7C). We also evaluated relationships between NCLDV vertical similarity and physical environmental factors including: the sampling depth of mesopelagic water, the mixed layer depth, and the temperature difference between epipelagic and mesopelagic waters. No significant correlations were detected among these parameters $(p>0.05, \mathrm{n}=32-36$ for surface samples and $\mathrm{n}=25-26$ for DCM samples) (Figs. 6D-F; Extended Data Figs. 7D-F).

243 We plotted correlations among the relative contributions of NCLDV phylotypes

244 between the euphotic and aphotic zones at all sampling locations (Extended Data Figs. 8 245 and 9). Where there was a strong similarity in the NCLDV community found at different 246 depths, Phycodnaviridae generally contributed highly to samples from the Arctic Ocean 247 (e.g., TARA stations 158, 201 and 209), and both Mimiviridae and Phycodnaviridae 248 contributed strongly in tropical and subtropical regions (e.g., stations 72, 110 and 122). 


\section{Discussion}

251 We investigated the diversity and community structure of NCLDVs based on

252 metagenomic PolB sequences collected from the world oceans. NCLDV communities

253 differed substantially between pico- and femto- size fractions (Fig. 1). NCLDV 254 communities in the pico-size fractions were dominated by Mimiviridae and 255 Phycodnaviridae, regardless of sampling location or depth (Fig. 1A). In marine 256 environments, species from the haptophytes (the genera Prymnesium, Haptolina, and 257 Phaeocystis), chlorophytes (Pyramimonas), pelagophytes (Aureococcus), bicosoecids 258 (Cafeteria) and choanoflagellates (Bicosta) are known hosts of Mimiviridae, while 259 species of haptophytes (Emiliania), chlorophytes (Ostreococcus, Micromonas and 260 Bathycoccus) and raphidophytes (Heterosigma) have been reported as Phycodnaviridae 261 hosts (Virus-Host DB) ${ }^{32}$. Although the dominance of Mimiviridae and Phycodnaviridae 262 have been reported in previous studies, mainly from coastal seawater ${ }^{13,14}$, our results 263 demonstrate the ubiquitous nature of these protist-infecting viruses across world ocean 264 biomes. It is worth noting that most of the NCLDVs (99.7\%) detected from the femtosize fraction were also present in the pico-size fraction (Fig. 3A), despite the large differences in relative abundance between two size fractions at each location. Therefore, the abundance information can be important for characterizing the differences of NCLDV communities. A proportion of the NCLDVs in the pico-size fraction were present within infected cells, because cell sizes of some host species such as Aureococcus anophagefferens and Micromonas pusilla are less than $3 \mu \mathrm{m}$. Thus, the abundance of

271 these lineages in the pico-size fraction may be partly enriched by the viruses replicating 272 inside their hosts.

273 In addition to Phycodnaviridae and Mimiviridae, Asfarviridae also contribute an 274 important proportion of NCLDVs in the femto-size fraction of most euphotic zones (Fig. 275 1B). Although very limited information is available regarding the natural hosts for this 276 group, a representative Asfarviridae-like species in marine environments is Heterocapsa circularisquama DNA virus (HcDNAV), which infects the red-tide-forming 
278 dinoflagellate $H$. circularisquama ${ }^{33}$. In the terrestrial ecosystem, this viral family is 279 known to infect a wide variety of organisms such as amoebozoa, arthropods and 280 mammals ${ }^{32,34}$. Given the broad range of host species for this viral lineage, there may be 281 an unknown but wide-spread host taxa for Asfarviridae in the ocean.

282 Our study revealed a heterogeneous pattern in the distribution of NCLDVs across the 283 oceans of the world (Fig. 2C). Although there are limited studies available on the factors controlling the large-scale distribution of viruses, it is widely accepted that both deterministic (environmental factors and inter-specific interactions) and stochastic processes (e.g., immigration and speciation) are important in making up microbial assemblages ${ }^{35-37}$. The distribution and diversity of viruses would not be directly affected by environmental variables such as temperature and nutrient availability, but is directly influenced by the geographic ranges of their host species ${ }^{3,38}$. Recent work with cyanophages demonstrated that a significant number of free-living viruses are locally produced through active infection rather than from migration ${ }^{39}$. Therefore, we expect that viral community structure will reflect host distribution as well as infectious activity.

Despite significant differences in community composition across oceanic biomes, we

294 found that most NCLDV phylotypes are dispersed throughout tropical and temperate 295 regions (Figs. 3A and 3B), presumably following their host community composition, 296 which is primarily determined by temperature ${ }^{40}$. However, the polar biome (mainly the 297 Arctic Ocean) constitutes a "hotspot" of unique NCLDV phylotypes from a wide variety 298 of families, despite having a low total richness in comparison to other regions (Figs. 3B 299 and 3C). We revealed that NCLDVs unique to non-polar biome were also abundant (Fig. 300 4), indicating a strong separation of NCLDV communities between polar and non-polar 301 biomes. A geographical barrier and steep environmental gradients may underlie this 302 distinct ecosystem structure (i.e., different host communities and their productivity) in the 303 Arctic Ocean ${ }^{27,28,31}$. Moreover, the Arctic Ocean is characterized by high amounts of river 304 discharge, contributing more than $10 \%$ to global runoff flux ${ }^{41}$. Consequently, biological 305 processes in the Arctic may be influenced by river inputs from terrestrial ecosystems. 
306 These factors may collectively contribute to the remarkable number of unique NCLDV 307 phylotypes found in the Arctic, that were undetectable in other regions. The biogeography 308 of NCLDVs on a global scale implies a tight link between the NCLDVs and the 309 distribution of their hosts, which is strongly influenced by physicochemical and 310 biological factors.

311 Tight coupling between NCLDVs and their hosts was further corroborated by our partial Mantel statistics, which described both known virus-host interactions and

313 additional but currently unrecognized associations between viruses and eukaryotic 314 lineages at the community level. Using the pico-sized NCLDV community, we detected 315 almost all known virus-host interactions, except for those involving Bicoecea (Fig. 5C). 316 This demonstrates that distance-based correlation analysis using global ocean samples is useful for detecting virus-host interplay in natural environments, although the validations

318 of the previously unknown associations remain to be further explored. Strong positive relationships between NCLDVs and eukaryotes involved many phytoplankton lineages including haptophytes, chlorophytes, dinophytes, pelagophytes and raphidophytes, all of which include known host lineages of NCLDVs (Fig. 5C). Strong correlations were also detected with heterotrophic choanoflagellates, which have recently been identified as a novel host of Mimiviridae ${ }^{25}$. Some NCLDVs, especially Mimiviridae, had strong correlations with chrysophytes, although no host species have yet been reported for this lineage. Many environmental NCLDV genomes were found to encode genes that are likely to be derived from marine chrysophytes (Supplementary Data 1-3). Taxonomic analyses based on PolB phylogeny and homology search revealed that most of these phylotypes represent previously unknown clades of the Mimiviridae tree (Extended Data 4 and 6; Supplementary Data 4), suggesting that chrysophytes may be an important host lineage of Mimiviridae in the ocean.

The global distribution of NCLDVs are determined by the geographic ranges of their 332 host organisms. Therefore, the virus-eukaryote associations that we detected likely arose under these constraints. On the other hand, it is expected that NCLDVs influence the 
334 abundance of eukaryotes at a local scale. Previous studies show that bacterial viruses have 335 an important role in determining bacterial mortality, because they substantially 336 outnumber their hosts and have highly specific infection mechanisms ${ }^{42}$. Similarly, 337 NCLDVs are reported to be more abundant than their host cells and have high infection specificity $^{11,14,43}$. For example, Emiliania huxleyi viruses (EhVs) of the Phycodnaviridae

339 family are responsible for almost all of the mortality of the haptophyte E. huxleyi during 340 blooms ${ }^{22,44,45}$. Another field study suggests that viral lysis can explain a greater proportion 341 of phytoplankton mortality than grazing by zooplankton ${ }^{6}$. These studies, combined with 342 the global associations that were detected in this study, emphasize the potential 343 importance of NCLDVs in structuring eukaryotic communities.

344 Our results indicate that marine phytoplankton lineages could represent one of the most important host groups of NCLDVs. Therefore, NCLDVs could be involved in the

346 regulation of biogeochemical processes mediated by phytoplankton. We investigated this 347 by assessing the vertical connectivity of viral communities. The NMDS analysis showed 348 clear differences between the NCLDV community composition of epipelagic (euphotic) 349 and mesopelagic (aphotic) zones at most sampling sites (Fig. 2B). Similar results were 350 also reported for phage communities in the Pacific Ocean ${ }^{46}$. The vertical separation of 351 viral communities may be caused by the stable stratification below the mixed layers 352 (typically above $200 \mathrm{~m}$ depth), which severely inhibits vertical water exchange. Despite 353 this limitation, mesopelagic ecosystems shared a significant number (98.7\%) of NCLDV 354 phylotypes with the upper epipelagic layers (Fig. 3A), suggesting the vertical connectivity 355 of NCLDVs and their local adaptation. Indeed, some mesopelagic NCLDV communities 356 were very similar to surface communities (Fig. 6A and Extended Data Fig. 7A). This 357 implies that the surface and mesopelagic NCLDV communities may be connected at some 358 locations. The major source of energy and materials in the mesopelagic layer is the 359 gravitational export of organic particles from the surface layer (i.e., the biological carbon 360 pump $)^{47-49}$. Therefore, some surface viruses may be exported to mesopelagic layers with sinking aggregated phytoplankton cells ${ }^{50-52}$. 
A significant positive correlation existed between surface phytoplankton biomass and NCLDV community similarity across depths (Fig. 6B and Extended Data Fig. 7B). Since highly productive areas are likely to have a greater flux of settling particles to the deep layers, this result supports the idea that NCLDVs are transported with the sinking particles. High vertical connectivity was consistently associated with an increase in NCLDV richness in the mesopelagic zone (Fig. 6C and Extended Data Fig. 7C). Previous studies showed that sinking particles can transfer bacterial and phage populations to the deep layer ${ }^{52,53}$. Mestre et al. $^{52}$ demonstrated that particle-attached prokaryotes had higher capacity for immigration than free-living ones. Based on the particle-driven vertical dispersion model, we can expect that NCLDVs, inside or attached to their host cells or cell debris, might be preferentially exported into the deep sea. Numerous studies based on sediment trap measurement have shown that larger phytoplankton, such as diatoms, contribute strongly to vertical flux because of their high sinking velocities ${ }^{54,55}$. However, recent studies show that smaller phytoplankton including haptophytes and chlorophytes, known hosts of marine NCLDVs, also contribute greatly to downward carbon export ${ }^{8,9,56}$. The high vertical connectivity of NCLDVs was not affected by the extent of the depth range nor by proxies for vertical mixing (Figs. 6D-F and Extended Data Figs. 7D-F), indicating that the migration of NCLDVs occurred regardless of physical processes such as upwelling, turbulent mixing, and convection. This result suggests that sinking export is a major source of a variety of NCLDVs to deeper waters, where NCLDV diversity is relatively low without this effect. A recent study revealed that some Phycodnaviridae and Mimiviridae potentially accelerate biological carbon export from the productive surface layer to deep layers, presumably by promoting cell death and aggregation of their host species $^{57}$. Phycodnaviridae and Mimiviridae also contributed strongly to high vertical connectivity in our study (Extended Data Figs. 8 and 9). The infection of the coccolithophore by the Phycodnaviridae EhV was observed to facilitate the sinking of host cells, likely by enhancing the production of transparent exopolymer particles and subsequent aggregation ${ }^{9}$. Therefore, the high vertical connectivity of NCLDVs detected 
390 in our analysis may be partly associated with enhanced vertical export of their infected 391 hosts.

392 The present study expands our knowledge of marine NCLDV biogeography. Most 393 NCLDV phylotypes are ubiquitously distributed over the oceans of the globe, although a 394 high proportion of unique NCLDVs was detected in the Arctic Ocean. Our comparison 395 of community distribution patterns highlighted the tight interplay between NCLDVs and 396 microeukaryotes. As marine ecological and biogeochemical processes are governed 397 primarily by microbes, NCLDVs would have an important influence on the dynamics of 398 marine systems. We also identified unexpected similarity of NCLDV communities 399 between surface and deep waters at some locations. This supports the idea that viral 400 activity may be related to the strength of the biological carbon pump, because the 401 efficiency and sinking rate of export production depends largely on surface phytoplankton 402 composition and their infection status ${ }^{8,9,55,58}$. Our findings underscore the importance of 403 NCLDVs as a component of marine microbial communities, and contribute to refine our 404 knowledge of marine ecosystems, a key regulator of the Earth's climate.

405

\section{Methods}

\section{Sample collection}

408 Metagenomic datasets were generated from samples collected by the Tara Oceans 409 expeditions from 2009 to $2013^{26-28,31,59}$. The second version of the Ocean Microbial 410 Reference Gene Catalog (OM-RGC.v2) is a non-redundant gene catalog constructed from 411370 metagenomic samples from the Tara Oceans project ${ }^{28}$ (https://www.ocean412 microbiome.org). The catalog includes 46,775,154 genes in total, and the gene abundance 413 profiles are expressed as the sum of within-reads aligned base pairs normalized by gene 414 length, in Tara Oceans samples ${ }^{28}$. 
418 polymerase (polB) as a marker gene of NCLDVs. Initially, amino acid sequences of the 419 OM-RGC.v2 were searched against an in-house profile hidden Markov model (HMM) of 420 NCLDV PolB sequences using the software HMMER, hmmsearch (version 3.1) ${ }^{60}$ with a 421 threshold E-value $<1 \times 10^{-5}$. Consequently, 29,315 PolB sequences were obtained from the 422 OM-RGC.v2, although this collection included sequences other than NCLDVs. To 423 remove the sequences not derived from NCLDVs and classify the taxonomic identity of each NCLDV sequence, phylogenetic mapping was performed within known PolB sequences. A maximum-likelihood (ML) reference phylogenetic tree was built based on 211 PolB reference protein sequences from eukaryotes, bacteria, archaea, phages and NCLDVs. These sequences were aligned using the default settings of the multiple sequence alignment program MAFFT-linsi (version 7) ${ }^{61}$ and ML tree was constructed with the use of randomized axelerated maximum likelihood (RAxML) program (version 7.2.8) ${ }^{62}$. In the reference trees, we included sequences from eight proposed families of NCLDVs ${ }^{63}$ Mimiviridae (synonymous with Megaviridae), Phycodnaviridae, Pithoviridae, Marseilleviridae, Ascoviridae, Iridoviridae, Asfarviridae, and Poxviridae (Extended Data Figs. 2-4). A sequence from a novel NCLDV clade Medusavirus was also included as a reference ${ }^{64}$. Query sequences were aligned against the reference alignment using the MAFFT 'addfragments' option, and then mapped onto the reference tree using the software program pplacer ${ }^{29}$.

\section{Abundance profiling of NCLDVs}

We used the abundance profile of NCLDV genes from the OM-RGC.v2 to evaluate 440 the relative frequency and diversity of NCLDVs. In the abundance matrix, we only 441 included samples from the pico-size $(0.22-1.6$ or $0.22-3.0 \mu \mathrm{m})$ and femto-size $(<0.22$ $442 \mu \mathrm{m})$ fractions. Samples used in the analysis were from three depth ranges: the surface (2$4439 \mathrm{~m}$ ), the deep chlorophyll maximum (DCM, 15-180 m) and the mesopelagic (MES, 250$4441,000 \mathrm{~m})$. The sum of length-normalized PolB abundances ranged from 5.3 to 22,847.5 across samples. The samples containing low PolB abundances tended to yield lower 
446 diversity estimates (i.e., number of phylotypes and Shannon’s entropy) (Extended Data

447 Fig. 10). To avoid bias due to the low sequencing effort, samples for which the sum of 448 length-normalized PolB abundance was less than 50 (set as a proxy for low NCLDV 449 frequency) were removed from the analysis. The abundance matrix was then standardized 450 by the sample with the lowest sum of length-normalized PolB abundance value. The 451 minimum value of PolB abundance among NCLDV phylotypes in the sample having the 452 lowest sum of length normalized PolB was set as the cutoff threshold. For each sample, 453 NCLDV phylotypes with a length-normalized abundance of less than this threshold were 454 treated as absent. A sample of a femto-size fraction of surface water from station 155 was 455 also removed, because it contained only one NCLDV PolB after standardization. 456 Consequently, our dataset was comprised of 283 samples (172 pico-fraction samples and 457111 femto-fraction samples), covering 88 sampling sites. These sites were categorized 458 into four biomes (coastal, trades, westerlies and polar biomes) according to latitude or 459 distance from the shore, and nine oceanic regions, as defined by Longhurst ${ }^{65}$ 460 (Supplementary Table 1).

\section{Phylogenetic tree construction}

463 To construct a phylogenetic tree, the NCLDV-derived PolB sequences obtained from 464 the OM-RGC.v2 were filtered by length ( $\geq 700$ amino acid sequences) because the 465 inclusion of short sequences yields unreliable phylogenies. Amino acid sequences from 466 the resulting 911 genes were aligned with known NCLDV sequences using the linsi 467 option from the MAFFT. The ML tree was constructed using RAxML with the use of a 468 known NCLDV sequence tree as a backbone constraint. We confirmed the validity of the 469 pplacer family assignment for 905 out of 911 selected sequences. The remaining six 470 sequences that were incorrectly placed within the phylogenetic tree were removed. The 471 ML tree was visualized using the program iTOL ${ }^{66}$. 
To explore the genomic contents of environmental NCLDVs, we made use of two sets of metagenome-assembled genomes (MAGs) of NCLDVs (GVMAGs high and medium quality $^{19}$; MoMAGs ${ }^{20}$ ), which were generated from environmental metagenomic datasets collected on global scales. Gene prediction was made for all MAGs using the program GeneMarkS ${ }^{67}$, then the predicted genes were searched using BLASTP against a database that combines the NCBI Reference Sequence database (RefSeq release 90) and the marine microbial eukaryote transcriptomes project (MMETSP) database ${ }^{68}$. We identified MAGs whose genes exhibited the best hit to transcripts of chrysophytes with $>50 \%$ amino acid identity and >100 alignment length (Supplementary Data 1). For these MAGs, we checked the redundancy between the MoMAG and GVMAG datasets using average nucleotide identity of $\geq 95 \%$ and an alignment fraction of $\geq 50 \%$ with FastANI (version 1.3) ${ }^{69}$. Although seven MAGs were found to be overlapped between the two datasets (Supplementary Data 1), all of the MAGs were retained for downstream analyses as these had different contig structures. The chrysophyte-related genes were considered potential candidates for horizontal gene transfer between chrysophytes and NCLDVs, and were BLASTP searched against the RefSeq database for additional functional annotation (Supplementary Data 2). We then extracted PolB sequences from the NCLDV MAGs which had a chrysophyte-related gene using the HMMER hmmsearch program. These PolBs were BLASTP searched against the NCLDV PolBs from the OM-RGC.v2. MAGderived PolBs aligned with over 700 amino acid sequences with $>90 \%$ identity were assigned to the PolB phylotypes derived from the OM-RGC.v2 (Supplementary Data 3). Phylogenetic affiliations of PolB from the chrysophyte-related MAGs were confirmed using a phylogenetic tree. To further test the credibility of our analysis, we checked other genes on the contigs that harbored the chrysophyte homologs using BLASTP against the RefSeq database (Supplementary Data 4; Extended Data Fig. 6).

\section{Diversity analyses}

501 Diversity and multivariate analyses were performed using the statistical software R 
502 (version 3.6.2) (https://www.r-project.org/). To evaluate the diversity of each sample, the 503 number of NCLDVs (richness) and Shannon's entropy were assessed by the package 504 'vegan’ (https://cran.r-project.org/web/packages/vegan). NCLDV richness among sizes 505 and depths were compared using a Kruskal-Wallis test followed by Dunn's multiple 506 comparison. Compositional variation among samples was assessed with a non-metric multidimensional scaling (NMDS) ordination based on Bray-Curtis dissimilarity. Statistical significance of differences among the sample groups (size, depth and biomes) was tested using a permutational multivariate analysis of variance (PERMANOVA) ${ }^{70}$ with 9,999 permutations.

\section{$512 \quad$ Partial Mantel test}

513 A partial Mantel test was performed to assess the correlation between two multivariate 514 matrices while controlling the potential effects of geographic distance (spatial 515 autocorrelation) using the R package 'vegan'. Abundance matrices for the NCLDV and 516 eukaryotic lineages were constructed from the integrated abundance tables, and the total 517 abundance at each site was normalized to 1 . The eukaryote abundance table was 518 constructed based on 18S rRNA gene metabarcoding ${ }^{71}$. Data for NLCDVs were obtained 519 from pico- $(0.22-1.6 / 3.0 \mu \mathrm{m})$ or femto-size $(<0.2 \mu \mathrm{m})$ fractions and for the eukaryotic 520 community from the pico- to meso-size fraction $(0.8-2,000 \mu \mathrm{m})$. There were 84 521 overlapping sampling events between pico-size NCLDVs and eukaryotic communities 522 and 55 overlapping sampling events between femto-size NCLDVs and eukaryotic 523 communities. All overlapping samples were derived from the surface or DCM depth 524 layers. Distance matrices for viruses and eukaryotes were calculated using the Bray525 Curtis measure. Geographic distances among sample sites were also measured using 526 Haversine distance and were used as a third distance matrix. Partial Mantel correlations 527 were computed between all pairs of distance matrices of eukaryotic communities and 528 NCLDVs with 9,999 permutations for each comparison. The false discovery rate (FDR) 529 was computed using the Benjamini-Hochberg method ${ }^{72}$. 


\section{Statistical test}

$532 \quad$ Two-sided test was applied for all statistical tests.

533

\section{Data availability}

535 The complete sequence data of the OM-RGC.v2 and the abundance profile can be 536 downloaded from https://www.ocean-microbiome.org. All sequences of 18S rRNA gene

537 metabarcoding have been deposited at European Nucleotide Archive (ENA) under the 538 BioProject ID PRJEB6610 and PRJEB9737. Environmental metadata are archived at 539 https://doi.pangaea.de/10.1594/PANGAEA.875582. Files used for recruiting NCLDV 540 PolB genes as well as processed abundance profiles of eukaryotes and NCLDVs with 541 corresponding environmental data are available at the GenomeNet FTP: 542 ftp://ftp.genome.jp/pub/db/community/tara/Biogeography/.

\section{Code availability}

545 Custom scripts developed for this study are available at GitHub: 546 https://github.com/HisashiENDO/NCLDV_Biogeography. 


\section{$548 \quad$ Figure legends}

549 Figure 1 Latitudinal patterns in NCLDV community composition. Relative contributions of NCLDV families at each depth range of (A) pico- and (B) femto-size fractions. The number of phylotypes detected in each sample is also indicated with a white circle. Sampling stations were arranged in rows from south to north, and color-coded based on biome (for a map of the sampling stations, please see Salazar et al., 2019²8).

Figure 2 Community characteristics of NCLDVs. Non-metric multidimensional scaling (NMDS) ordination based on the NCLDV community showing results for all samples (A) and separately for pico- and femto-size fractions (B and C). Sample groups are color-coded by size fraction (A), depth (B) and biome (C). Ellipses represent $90 \%$ confidence levels for each group. All group categories are significantly different from each other as analyzed using PERMANOVA ( $p$ $<0.01)$. Sample sizes for the test are noted in Supplementary Table 1.

Figure 3 Structural differentiation of NLCDV community across ecological zones.

(A) Venn diagrams showing the numbers of shared or unique NLCDVs phylotypes across size fractions (left), depths (center) and biomes (right). (B) Map showing the number of total, unique and shared NCLDVs across nine oceanic regions. The map was drawn using the $\mathrm{R}$ package 'maps' (https://cran.r-project.org/web/packages/maps). (C) Relationships among sample size and total or unique NCLDVs detected in each region. Abbreviations: SO: Southern Ocean; RS: Red Sea; MS: Mediterranean Sea; NPO: North Pacific Ocean; NAO: North Atlantic Ocean; SAO: South Atlantic Ocean; SPO: South Pacific Ocean; IO: Indian Ocean; AO: Arctic Ocean.

Figure 4 Phylogenetic affiliations of environmental NCLDVs and their dispersal characteristics. Phylogenetic tree constructed from 905 long $(\geq 700$ amino acid) PolB sequences from the OM-RGC.v2 and 67 known NCLDV sequences (see also Extended Data Figs. 2-4 for details). The first six layers indicate the occurrence of NCLDVs unique to each size fraction, depth and biome. The outside layer denotes phylogenetic positions of known sequences (color code as in the legend) and the phylotypes closely related ( $>90 \%$ amino acid identity) to those of NCLDV MAGs having chrysophyte homologs (indicated in yellow). Abbreviations: OLPV-2: Organic Lake phycodnavirus 2; OLPV-1: Organic Lake phycodnavirus 1; CeV: Chrysochromulina ericina virus 1; PgV: Phaeocystis globosa virus 16T; HeV: Haptolina ericina virus RF02; PkV-2; Prymnesium kappa virus RF02; TetV-1: Tetraselmis virus 1; PoV: Pyramimonas orientalis virus 1; AaV: Aureococcus anophagefferens virus BtV-01; PkV-1; Prymnesium kappa virus RF01; ChoanoV: ChoanoVirus; CroV: Cafeteria roenbergensis virus BV-PW1; MpV-1: Micromonas sp. 
587

588

589

590

591

592

593

594

595

596

597

598

599

600

601

602

603

604

605

606

607

608

609

610

611

612

613

614

615

RCC1109 virus MpV1; OlV-1: Ostreococcus lucimarinus virus 1; Otv-1: Ostreococcus tauri virus 1; Otv-2: Ostreococcus tauri virus 2; MpV-12T: Micromonas pusilla virus 12T: BpV-1: Bathycoccus sp. RCC1105 virus; BCVFR483: Paramecium bursaria Chlorella virus FR-483; ACTV-1: Acanthocystis turfacea Chlorella virus 1; PBCV-1: Paramecium bursaria Chlorella virus 1; EhV-86: Emiliania huxleyi virus 86; FsV: Feldmannia species virus; EsV-1: Ectocampus siliculou virus 1; P. salinus: Pandoravirus salinus; P. dulcis: Pandoravirus dulcis; HaV-1: Heterosigma akashiwo virus 1.

Figure 5 Associations between NCLDVs and eukaryotic communities. (A) Partial Mantel correlation coefficients (Spearman's $\rho$ ) between NCLDVs and eukaryotic communities. Each plot shows the value of $\rho$ computed based on pico- (x-axis) and femto-sized (y-axis) NCLDV communities. Known virushost associations are shown as red dots. (B) Histogram and density estimates showing the distribution of $\rho$ values in known (red) and unknown (gray) pairs. (C) Pairwise comparisons of the partial Mantel correlation coefficients between NCLDV and eukaryotic lineages. Correlation coefficients $\rho>0.53$ based on pico-size NCLDV communities are drawn as edges. Known virus-host associations are shown in red, whereas unknown associations are shown in gray.

Figure 6 Vertical linkage of NCLDV communities between the surface and mesopelagic layers. (A) Latitudinal trend in NCLDV community similarity between two depths (with the station numbers). Relationship between NCLDV vertical similarity and (B) the surface chlorophyll $a$ biomass, (C) NCLDV richness in the mesopelagic layer, (D) sampling depth of mesopelagic seawater, (E) the mixed layer depth and (F) temperature difference between epipelagic and mesopelagic samples. All NCLDV data were generated based on the picosize fraction. Shaded areas represent $90 \%$ confidence intervals. 


\section{References}

6171 Field, C. B., Behrenfeld, M. J., Randerson, J. T. \& Falkowski, P. Primary 618 production of the biosphere: integrating terrestrial and oceanic components. $619 \quad$ Science 281, 237-240, doi:10.1126/science.281.5374.237 (1998).

6202 Worden, A. Z. et al. Environmental science. Rethinking the marine carbon cycle: 621 factoring in the multifarious lifestyles of microbes. Science 347, 1257594, 622 doi:10.1126/science.1257594 (2015).

6233 Brum, J. R. \& Sullivan, M. B. Rising to the challenge: accelerated pace of 624 discovery transforms marine virology. Nat Rev Microbiol 13, 147-159, 625 doi:10.1038/nrmicro3404 (2015).

6264 Selosse, M.-A., Charpin, M. \& Not, F. Mixotrophy everywhere on land and in 627 water: the grand écart hypothesis. Ecology Letters 20, 246-263, 628 doi:10.1111/ele.12714 (2017).

6295 Weitz, J. S. et al. A multitrophic model to quantify the effects of marine viruses 630 on microbial food webs and ecosystem processes. Isme $j$ 9, 1352-1364, 631 doi:10.1038/ismej.2014.220 (2015).

6326 Mojica, K. D., Huisman, J., Wilhelm, S. W. \& Brussaard, C. P. Latitudinal 633 variation in virus-induced mortality of phytoplankton across the North Atlantic 634 Ocean. Isme j 10, 500-513, doi:10.1038/ismej.2015.130 (2016).

6357 Suttle, C. A. Marine viruses--major players in the global ecosystem. Nat Rev 636 Microbiol 5, 801-812, doi:10.1038/nrmicro1750 (2007).

6378 Guidi, L. et al. Plankton networks driving carbon export in the oligotrophic ocean. $638 \quad$ Nature 532, 465-470, doi:10.1038/nature16942 (2016).

6399 Laber, C. P. et al. Coccolithovirus facilitation of carbon export in the North $640 \quad$ Atlantic. Nat Microbiol 3, 537-547, doi:10.1038/s41564-018-0128-4 (2018).

64110 Colson, P. et al. "Megavirales", a proposed new order for eukaryotic 642 nucleocytoplasmic large DNA viruses. Arch Virol 158, 2517-2521, 643 doi:10.1007/s00705-013-1768-6 (2013). 
64411 Fischer, M. G. Giant viruses come of age. Curr Opin Microbiol 31, 50-57, 645 doi:10.1016/j.mib.2016.03.001 (2016).

64612 Koonin, E. V. \& Yutin, N. Evolution of the Large Nucleocytoplasmic DNA 647 Viruses of Eukaryotes and Convergent Origins of Viral Gigantism. Adv Virus Res 648 103, 167-202, doi:10.1016/bs.aivir.2018.09.002 (2019).

64913 Monier, A., Claverie, J. M. \& Ogata, H. Taxonomic distribution of large DNA 650 viruses in the sea. Genome Biol 9, R106, doi:10.1186/gb-2008-9-7-r106 (2008).

65114 Hingamp, P. et al. Exploring nucleo-cytoplasmic large DNA viruses in Tara 652 Oceans microbial metagenomes. ISME J 7, 1678-1695, 653 doi:10.1038/ismej.2013.59 (2013).

65415 Clerissi, C. et al. Deep sequencing of amplified Prasinovirus and host green algal 655 genes from an Indian Ocean transect reveals interacting trophic dependencies and 656 657 new genotypes. Environ Microbiol Rep 7, 979-989, doi:10.1111/1758-2229.12345

65816 Li, Y. et al. The Earth Is Small for "Leviathans": Long Distance Dispersal of Giant 659 Viruses across Aquatic Environments. Microbes Environ 34, 334-339, 660 doi:10.1264/jsme2.ME19037 (2019).

66117 Mihara, T. et al. Taxon Richness of "Megaviridae" Exceeds those of Bacteria and 662 Archaea in the Ocean. Microbes Environ 33, 162-171, 663 doi:10.1264/jsme2.ME17203 (2018).

66418 Li, Y. et al. Degenerate PCR Primers to Reveal the Diversity of Giant Viruses in 665 Coastal Waters. Viruses 10, 496, doi:10.3390/v10090496 (2018).

66619 Schulz, F. et al. Giant virus diversity and host interactions through global 667 metagenomics. Nature, doi:10.1038/s41586-020-1957-x (2020).

66820 Moniruzzaman, M., Martinez-Gutierrez, C. A., Weinheimer, A. R. \& Aylward, F. 669 O. Dynamic genome evolution and complex virocell metabolism of globally670 distributed giant viruses. Nat Commun 11, 1710, doi:10.1038/s41467-020-15507671 $2(2020)$. 
67221 Cottrell, M. T. \& Suttle, C. A. Wide-spread occurrence and clonal variation in 673 viruses which cause lysis of a cosmopolitan, eukaryotic marine phytoplankter, $674 \quad$ Micromonas pusilla. Mar Ecol Prog Ser 78 (1991).

67522 Bratbak, G., Egge, J. K. \& Heldal, M. Viral mortality of the marine alga Emiliania 676 huxleyi (Haptophyceae) and termination of algal blooms. Marine Ecology $677 \quad$ Progress Series 93, 39-48 (1993).

67823 Kenji, T., Keizo, N., Shigeru, I. \& Mineo, Y. Isolation of a virus infecting the 679 novel shellfish-killing dinoflagellate Heterocapsa circularisquama. Aquatic $680 \quad$ Microbial Ecology 23, 103-111 (2001).

68124 Fischer, M. G., Allen, M. J., Wilson, W. H. \& Suttle, C. A. Giant virus with a 682 remarkable complement of genes infects marine zooplankton. Proc Natl Acad Sci 683 U S A 107, 19508-19513, doi:10.1073/pnas.1007615107 (2010).

68425 Needham, D. M. et al. A distinct lineage of giant viruses brings a rhodopsin 685 photosystem to unicellular marine predators. Proc Natl Acad Sci U S A 116, 686 20574-20583, doi:10.1073/pnas.1907517116 (2019).

68726 Pesant, S. et al. Open science resources for the discovery and analysis of Tara $688 \quad$ Oceans data. Sci Data 2, 150023, doi:10.1038/sdata.2015.23 (2015).

68927 Gregory, A. C. et al. Marine DNA Viral Macro- and Microdiversity from Pole to $690 \quad$ Pole. Cell 177, 1109-1123 e1114, doi:10.1016/j.cell.2019.03.040 (2019).

69128 Salazar, G. et al. Gene Expression Changes and Community Turnover 692 Differentially Shape the Global Ocean Metatranscriptome. Cell 179, 1068-1083 693 e1021, doi:10.1016/j.cell.2019.10.014 (2019).

69429 Matsen, F. A., Kodner, R. B. \& Armbrust, E. V. pplacer: linear time maximum695 likelihood and Bayesian phylogenetic placement of sequences onto a fixed 696 reference tree. BMC Bioinformatics 11, 538, doi:10.1186/1471-2105-11-538 697 (2010).

69830 Gallot-Lavallee, L., Blanc, G. \& Claverie, J. M. Comparative Genomics of 699 Chrysochromulina Ericina Virus and Other Microalga-Infecting Large DNA 
700 Viruses Highlights Their Intricate Evolutionary Relationship with the Established

701 Mimiviridae Family. J Virol 91, doi:10.1128/jvi.00230-17 (2017).

70231 Ibarbalz, F. M. et al. Global Trends in Marine Plankton Diversity across

703 Kingdoms of Life. Cell 179, 1084-1097 e1021, doi:10.1016/j.cell.2019.10.008

704 (2019).

70532 Mihara, T. et al. Linking Virus Genomes with Host Taxonomy. Viruses 8, 66, 706 doi:10.3390/v8030066 (2016).

70733 Ogata, H. et al. Remarkable sequence similarity between the dinoflagellate708 infecting marine girus and the terrestrial pathogen African swine fever virus. Virol 709 J 6, 178, doi:10.1186/1743-422X-6-178 (2009).

71034 Andreani, J. et al. Pacmanvirus, a New Giant Icosahedral Virus at the Crossroads

711 between Asfarviridae and Faustoviruses. J Virol 91, doi:10.1128/JVI.00212-17

712 (2017).

71335 Barton, A. D., Dutkiewicz, S., Flierl, G., Bragg, J. \& Follows, M. J. Patterns of 714 diversity in marine phytoplankton. Science 327, 1509-1511, 715 doi:10.1126/science.1184961 (2010).

$71636 \quad$ Lima-Mendez, G. et al. Ocean plankton. Determinants of community structure in 717 the global plankton interactome. Science 348, 1262073, 718 doi:10.1126/science.1262073 (2015).

71937 Zhou, J. \& Ning, D. Stochastic Community Assembly: Does It Matter in 720 Microbial Ecology? Microbiol Mol Biol Rev 81, doi:10.1128/mmbr.00002-17 (2017).

72238 Chow, C. E. \& Suttle, C. A. Biogeography of Viruses in the Sea. Annu Rev Virol $723 \quad$ 2, 41-66, doi:10.1146/annurev-virology-031413-085540 (2015).

$72439 \quad$ Yoshida, T. et al. Locality and diel cycling of viral production revealed by a $24 \mathrm{~h}$ 725 time course cross-omics analysis in a coastal region of Japan. Isme j 12, 1287726 1295, doi:10.1038/s41396-018-0052-x (2018).

72740 Sunagawa, S. et al. Ocean plankton. Structure and function of the global ocean 
728

$72941 \quad$ Syed, T. H., Famiglietti, J. S., Zlotnicki, V. \& Rodell, M. Contemporary estimates

microbiome. Science 348, 1261359, doi:10.1126/science.1261359 (2015). of Pan-Arctic freshwater discharge from GRACE and reanalysis. Geophysical

731

73242 Research Letters 34, doi:10.1029/2007gl031254 (2007).

733

$734 \quad 43$

735

736

737

738

739

740

741

$742 \quad 46$

743

744

745

746

Wommack, K. E. \& Colwell, R. R. Virioplankton: viruses in aquatic ecosystems. Microbiol Mol Biol Rev 64, 69-114, doi:10.1128/mmbr.64.1.69-114.2000 (2000). Bellec, L. et al. Cophylogenetic interactions between marine viruses and eukaryotic picophytoplankton. BMC Evol Biol 14, 59, doi:10.1186/1471-214814-59 (2014).

Brussaard, C. P. D., Kempers, R. S., Kop, A. J., Riegman, R. \& Heldal, M. Viruslike particles in a summer bloom of Emiliania huxleyi in the North Sea. Aquatic Microbial Ecology 10, 105-113 (1996).

45 Stephan, J. et al. Flow cytometric analysis of an Emiliana huxleyi bloom terminated by viral infection. Aquatic Microbial Ecology 27, 111-124 (2002).

Hurwitz, B. L., Westveld, A. H., Brum, J. R. \& Sullivan, M. B. Modeling ecological drivers in marine viral communities using comparative metagenomics and network analyses. Proc Natl Acad Sci U S A 111, 10714-10719, doi:10.1073/pnas.1319778111 (2014).

Herndl, G. J. \& Reinthaler, T. Microbial control of the dark end of the biological pump. Nat Geosci 6, 718-724, doi:10.1038/ngeo1921 (2013).

Giering, S. L. et al. Reconciliation of the carbon budget in the ocean's twilight zone. Nature 507, 480-483, doi:10.1038/nature13123 (2014).

Boyd, P. W., Claustre, H., Levy, M., Siegel, D. A. \& Weber, T. Multi-faceted particle pumps drive carbon sequestration in the ocean. Nature 568, 327-335, doi:10.1038/s41586-019-1098-2 (2019).

Janice, E. L. \& Curtis, A. S. Effect of viral infection on sinking rates of Heterosigma akashiwo and its implications for bloom termination. Aquatic Microbial Ecology 37, 1-7 (2004). 
75651 Close, H. G. et al. Export of submicron particulate organic matter to mesopelagic 757 depth in an oligotrophic gyre. Proc Natl Acad Sci U S A 110, 12565-12570, $758 \quad$ doi:10.1073/pnas.1217514110 (2013).

75952 Mestre, M. et al. Sinking particles promote vertical connectivity in the ocean 760 microbiome. Proc Natl Acad Sci $U$ S A 115, E6799-E6807, $761 \quad$ doi:10.1073/pnas.1802470115 (2018).

76253 Hurwitz, B. L., Brum, J. R. \& Sullivan, M. B. Depth-stratified functional and 763 taxonomic niche specialization in the 'core' and 'flexible' Pacific Ocean Virome. 764 Isme j 9, 472-484, doi:10.1038/ismej.2014.143 (2015).

76554 Sancetta, C., Villareal, T. \& Falkowski, P. Massive fluxes of rhizosolenid diatoms: 766 A common occurrence? Limnology and Oceanography 36, 1452-1457, 767 doi:10.4319/lo.1991.36.7.1452 (1991).

76855 Kawakami, H. \& Honda, M. C. Time-series observation of POC fluxes estimated 769 from 234Th in the northwestern North Pacific. Deep Sea Research Part I: Oceanographic Research Papers 54, 1070-1090, doi:10.1016/j.dsr.2007.04.005 771 (2007).

$77256 \quad$ Richardson, T. L. \& Jackson, G. A. Small phytoplankton and carbon export from 773 the surface ocean. Science 315, 838-840, doi:10.1126/science.1133471 (2007).

77457 Blanc-Mathieu, R. et al. Viruses of the eukaryotic plankton are predicted to 775 776 increase carbon export efficiency in the global sunlit ocean. bioRxiv, 710228, doi:10.1101/710228 (2019).

77758 Iversen, M. H. \& Ploug, H. Ballast minerals and the sinking carbon flux in the 778 ocean: carbon-specific respiration rates and sinking velocity of marine snow aggregates. Biogeosciences 7, 2613-2624, doi:10.5194/bg-7-2613-2010 (2010).

78059 Alberti, A. et al. Viral to metazoan marine plankton nucleotide sequences from 781 the Tara Oceans expedition. Sci Data 4, 170093, doi:10.1038/sdata.2017.93 782 (2017).

78360 Eddy, S. R. Profile hidden Markov models. Bioinformatics 14, 755-763, 
784

785

786

787

788

789

790

791

792

$793 \quad 6$

794

$795 \quad 65$

796

$797 \quad 66$

798

799

800

801

802

803

$804 \quad 68$

805

806

807

808

809

810

$811 \quad 70$

doi:10.1093/bioinformatics/14.9.755 (1998).

61 Katoh, K. \& Standley, D. M. MAFFT multiple sequence alignment software version 7: improvements in performance and usability. Mol Biol Evol 30, 772-780, doi:10.1093/molbev/mst010 (2013).

62 Stamatakis, A. RAxML-VI-HPC: maximum likelihood-based phylogenetic analyses with thousands of taxa and mixed models. Bioinformatics 22, 2688-2690, doi:10.1093/bioinformatics/btl446 (2006).

63 Koonin, E. V. \& Yutin, N. Multiple evolutionary origins of giant viruses. F1000Res 7, doi:10.12688/f1000research.16248.1 (2018).

4 Yoshikawa, G. et al. Medusavirus, a Novel Large DNA Virus Discovered from Hot Spring Water. J Virol 93, doi:10.1128/JVI.02130-18 (2019).

5 Longhurst, A. R. in Ecological Geography of the Sea (Second Edition) (ed Alan R. Longhurst) 89-102 (Academic Press, 2007).

6 Letunic, I. \& Bork, P. Interactive tree of life (iTOL) v3: an online tool for the display and annotation of phylogenetic and other trees. Nucleic Acids Res 44, W242-245, doi:10.1093/nar/gkw290 (2016).

7 Besemer, J., Lomsadze, A. \& Borodovsky, M. GeneMarkS: a self-training method for prediction of gene starts in microbial genomes. Implications for finding sequence motifs in regulatory regions. Nucleic Acids Res 29, 2607-2618, doi:10.1093/nar/29.12.2607 (2001).

Keeling, P. J. et al. The Marine Microbial Eukaryote Transcriptome Sequencing Project (MMETSP): illuminating the functional diversity of eukaryotic life in the oceans through transcriptome sequencing. PLoS Biol 12, e1001889, doi:10.1371/journal.pbio.1001889 (2014).

69 Jain, C., Rodriguez, R. L., Phillippy, A. M., Konstantinidis, K. T. \& Aluru, S. High throughput ANI analysis of 90K prokaryotic genomes reveals clear species boundaries. Nat Commun 9, 5114, doi:10.1038/s41467-018-07641-9 (2018).

Anderson, M. J. A new method for non-parametric multivariate analysis of 

(2001).

81471 de Vargas, C. et al. Ocean plankton. Eukaryotic plankton diversity in the sunlit $815 \quad$ ocean. Science 348, 1261605, doi:10.1126/science.1261605 (2015).

81672 Benjamini, Y. \& Hochberg, Y. Controlling the False Discovery Rate: A Practical 817 and Powerful Approach to Multiple Testing. Journal of the Royal Statistical Society: Series B (Methodological) 57, 289-300, doi:10.1111/j.25176161.1995.tb02031.x (1995).

820

\section{Acknowledgement}

822 This work was supported by JSPS/KAKENHI (Nos. 26430184, 18H02279, and $82319 H 05667$ to H.O. and Nos. 19K15895 and 19H04263 to H.E.), Scientific Research on 824 Innovative Areas from the Ministry of Education, Culture, Science, Sports and 825 Technology (MEXT) of Japan (Nos. 16H06429, 16K21723, and 16H06437 to H.O.), 826 Kyoto University Research Coordination Alliance (funding to H.E.), and the 827 Collaborative Research Program of the Institute for Chemical Research, Kyoto University 828 (Nos. 2019-30 and 2020-27). Computational time was provided by the SuperComputer 829 System, Institute for Chemical Research, Kyoto University. We further thank the Tara 830 Oceans consortium, the projects OCEANOMICS (ANR-11-BTBR-0008) and France 831 Genomique (ANR-10-INBS-09), and the people and sponsors who supported Tara 832 Oceans. Tara Oceans (that includes both the Tara Oceans and Tara Oceans Polar Circle 833 expeditions) would not exist without the leadership of the Tara Expeditions Foundation 834 and the continuous support of 23 institutes (https://oceans.taraexpeditions.org). This 835 article is contribution number 108 of Tara Oceans.

836

\section{Author contributions}

838 HE and HO designed the study. HE performed most of the bioinformatics analysis. 839 RB-M and YL contributed to the bioinformatics analysis. GS, NH, KL, CdV, MBS, CB, 840 PW, LK-B, and SS contributed to the generation of primary data. CdV, MBS, CB, PW, 
841 LK-B, SS, and HO coordinated Tara Oceans. All authors contributed to the writing of the 842 manuscript.

843

\section{Materials \& Correspondence}

845 Correspondence and material requests should be addressed to HO (email:

846 ogata@kuicr.kyoto-u.ac.jp).

847

\section{Competing financial interests}

849 The authors declare no competing financial interests.

850

851 




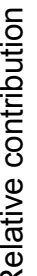
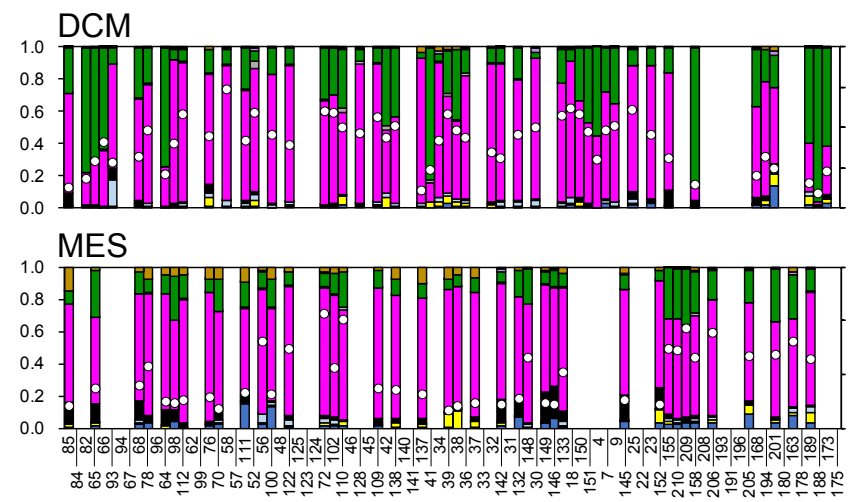

$\begin{array}{lll}-60-45 & -30 & -15\end{array}$

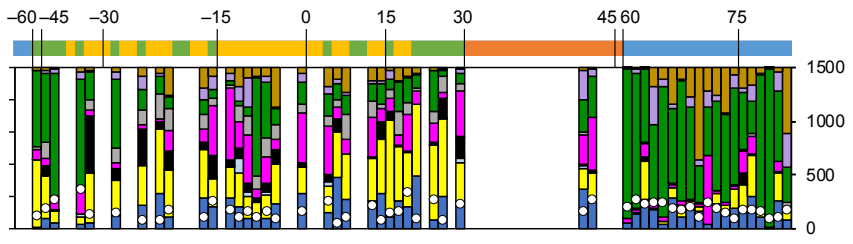

DCM

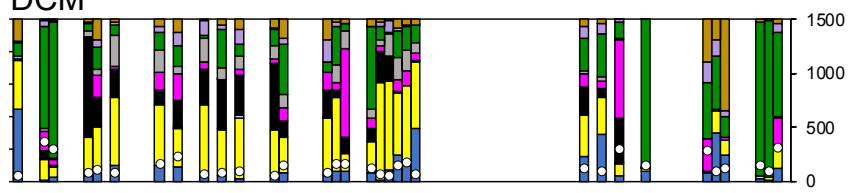

MES



Station number

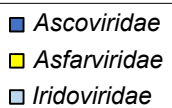

- Medusavirus

口 Phycodnaviridae

$\square$ Iridoviridae

$\square$ Mimiviridae

$\square$ Pithoviridae

$\square$ Marseilleviridae

$\square$ Poxviridae 


\section{都人学} Pico-SIZE KYOTO UNIVERSITY $(0.22-1.6 / 3.0 \mu \mathrm{m})$

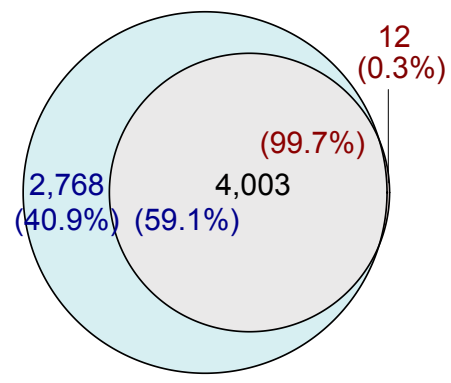

A Self-archived copy in
Kyoto University Research Information Repository https://repository.kulib.kyoto-u.ac.jp

Euphotic zone

(Surface and DCM) Mesopelagic zone



Non-Polar biome

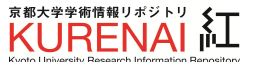

(Trades, Westerlies, and Coastal)

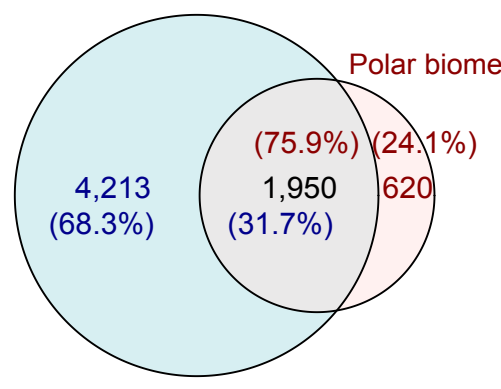

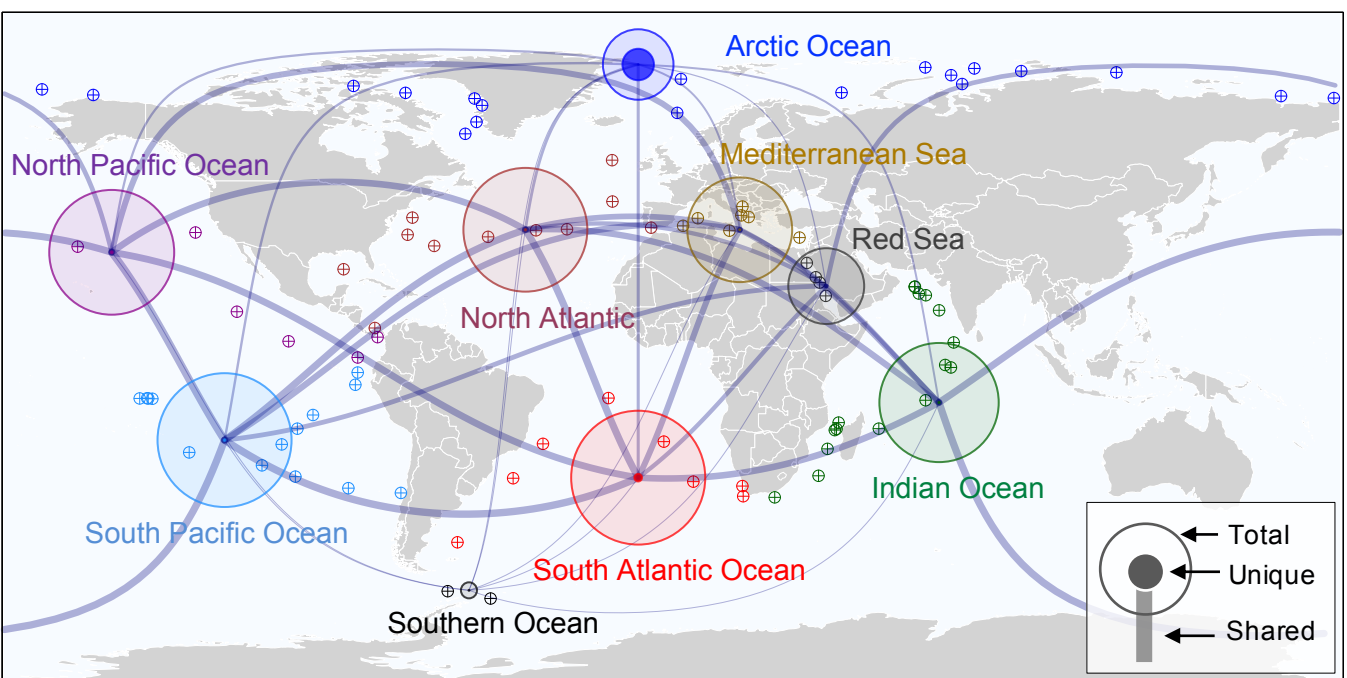
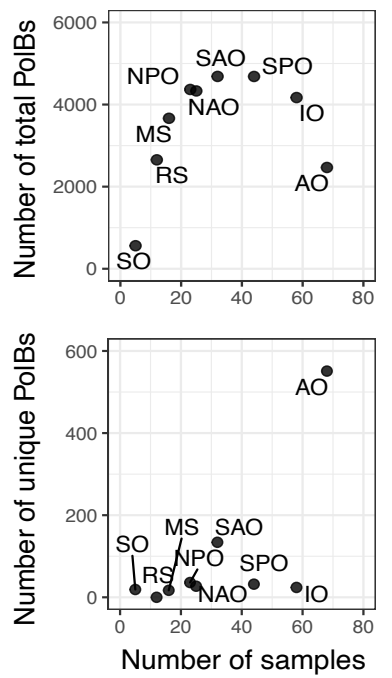
京都大学

A Self-archived copy in

京都大学学術情報リポジトリく

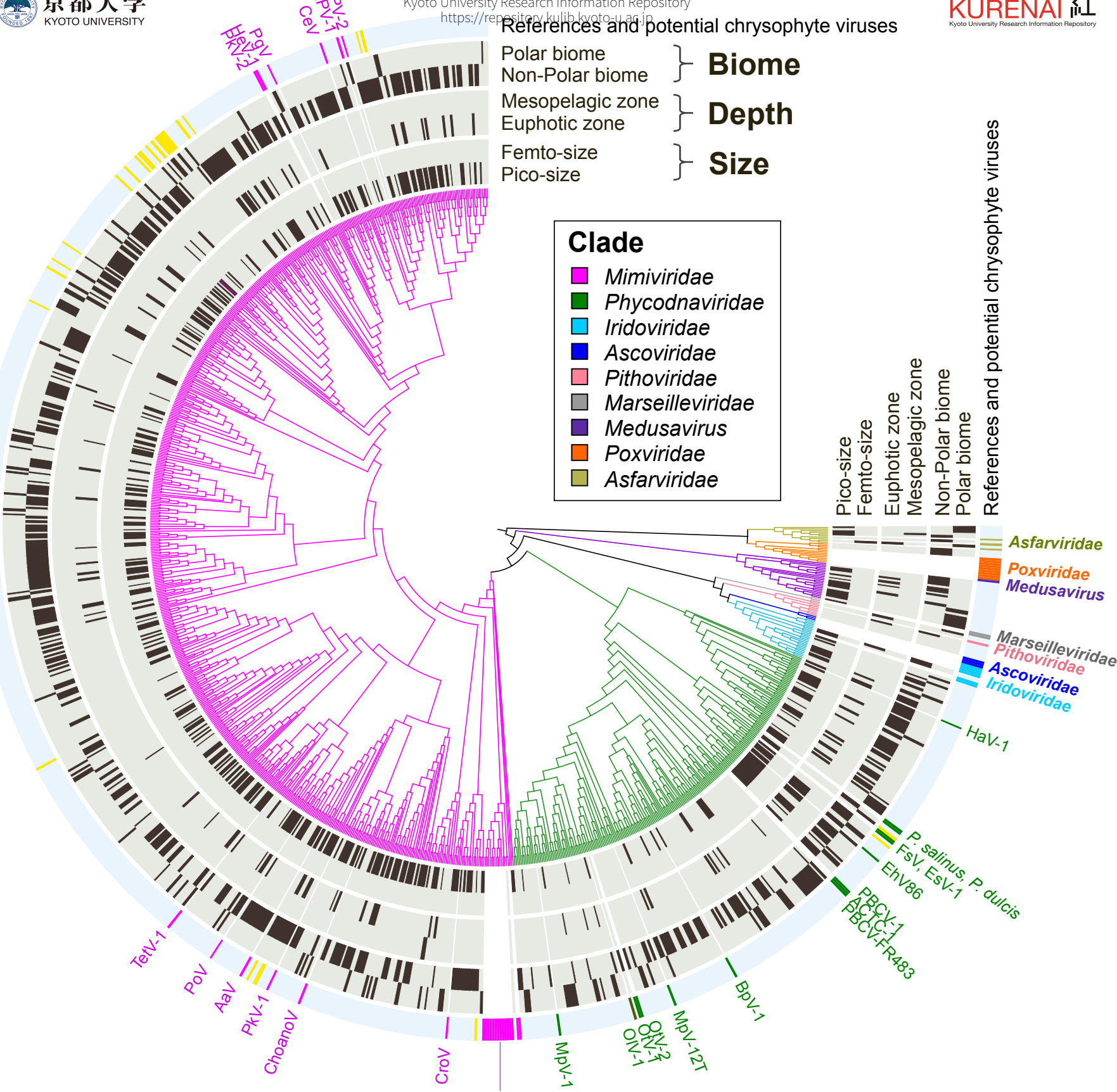


CAself-archived copy in

Bicoecea

京都大学学術情報リポジトリ
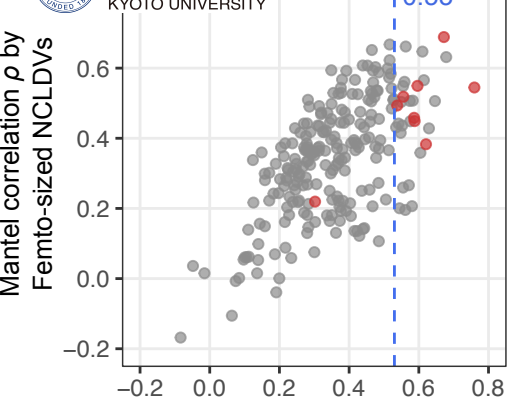

Symbiotic relationship

- Known

- Unknown
B



है

Mantel correlation $\rho$ by Pico-sized NCLDVs

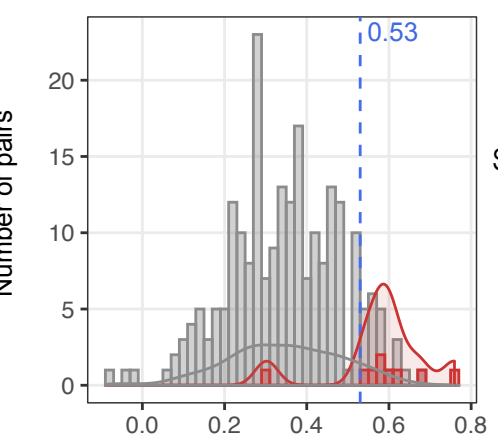

Symbiotic relationship

Known

Unknown
Category

NCLDV

Phototroph

Heterotroph ymbiotic relationship

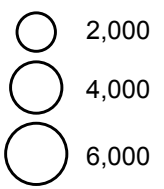

Correlation coefficient

0.55

$-0.60$

0.70 


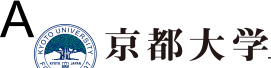

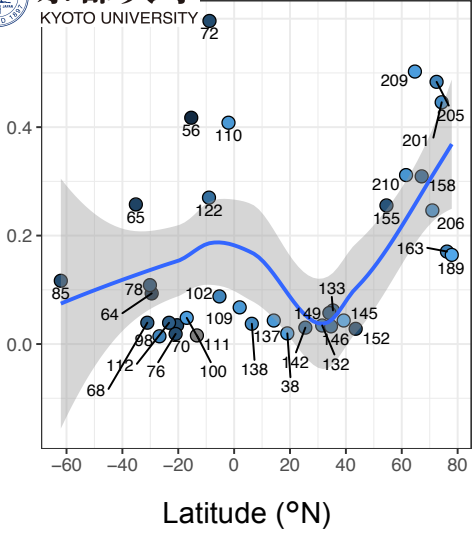

D

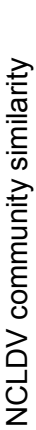

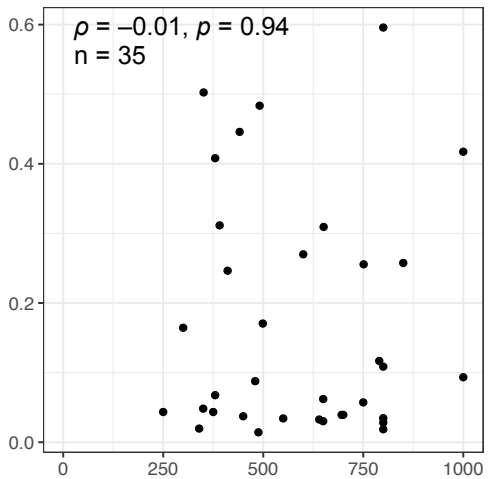

Depth of MES sample (m)
B A Self-archived copy in

Kyoto University Research Information Repository

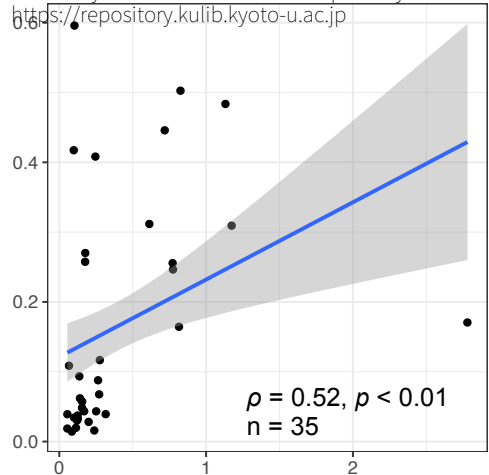

Surface chlorophyll a $\left(\mu g L^{-1}\right)$

E

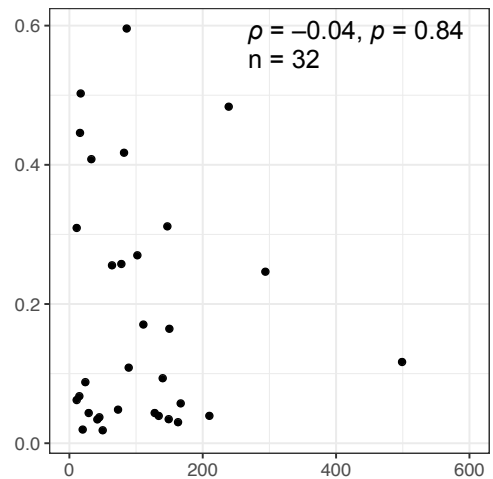

Mixed layer depth (m)

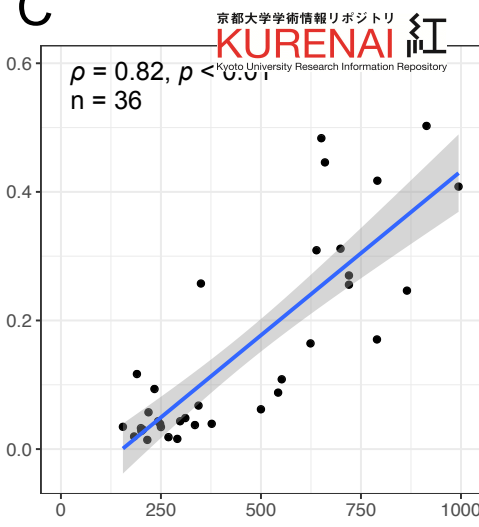

NCLDV richness in MES layer

F



Temperature difference between two sample depths $\left({ }^{\circ} \mathrm{C}\right)$ 